\title{
ACRL Midwinter meetings in Washington, D.C.
}

A tentative schedule for the ALA Midwinter Meeting in Washington, D.C., February 14-19, 1997

$\mathbf{a}$ d. note: This list was current as we went to press. Be sure to check the program book for final times and locations of meetings.

\section{ACRL Board of Directors}

Board/Leadership Council: Friday, Feb. 14, 2:00-4:00 p.m.

First meeting: Sunday, Feb. 16, 2:00-4:00 p.m. Joint meeting with Budget \& Finance Committee: Monday, Feb, 17, 11:00 a.m.-12:30 p.m.

Second meeting: Tuesday, Feb. 18, 2:00-5:30 p.m.

\section{ACRL General}

ACRI Presidential Candidates Forum and Reception: Saturclay, Feb. 15, 12:30-2:00 P.m.

Board/Leadership Council: Friday, Feb. 14, 2:00-4:00 p.m.

\section{ACRL Divisional Committees}

Academic/Research Librarian of the Year Award: Monday, Feb. 17, 8:00-9:00 a.m. (closed)

Academic Status: Saturday, Feb. 15. 8:30-11:00 a.m.; Sunday, February 16, 8:30-11:00 a.m.

Budget and Finance: Saturday, Feb. 15 , 8:30 a.m.-12:30 p.m.; Sunday, Feb. 16, 8:30 a.m.-12:30 p.m.; Monday, Feb. 17, 8:30 a.m.-12:30 p.m.; Tuesday, Feb. 18, 9:30 a.m. $-12: 30$ p.m.

Colleagues: Tuesday, Feb. 18, 9:30-11:00 a.m.

Community Information Organizations: Sunday, Feb. 16, 4:45-6:00 p.m.

Conference Program Planning Washington, D.C., 1998: Friday, Feb. 14, 12:30-2.00 p.m.
Conference Program Planning -San Francisco, 1997: Saturclay, Feb. 15, 8:30-11:00 a.m.

Constitution and Bylaws: Saturday, Feb.15, 11:30 a.m.-12:30 p.m.; Monday, Feb. 17, 11:30 a.m.-12:30 p.m.

Copyright: Sunday, Feb. 16, 8:30 a.m. $-12: 30$ p.m.

Doctoral Dissertation Fellowship: Saturday, Feb. 15, 9:30 a.m.-12:30 p.m.; Sunday, Feb. 16, 2:00-4:00 p.m. (closed)

Government Relations: Monday, Feh. 17 , 8:00-11:00 a.m.

Institutional Priorities and Faculty Rewards Task Force: Saturday, Feb. 15 , 4:30-5:30 p.m. Discussion: Saturday, Feb. 15 2:00-4:00 p.m

Intellectual Freedom: Saturday, Feb. 15, 9:30 a.m.-12:30 p.m.

International Relations: Sunday, Feb. 16, 8:30-11:00 a.m.

K.G. Saur Award: Saturday, Feb. 15, 8:30-11:00 a.m. (closed)

Lazerow Fellowship for Research in Acquisitions: Monday, Feb. 17, 8:00-11:00 a.m. (closed)

Leadership Center Advisory: Sunday, Feb. 16, 9:30-11:00 a.m

Media Resources: Sunday, Feb, 16 , 9:30-11:00 a.m.

Membership: Sunclay, Feb. 16, 4:30-5:30 p.m.

National Conference-Nashville: Saturday, Feb. 15, 8:30-12:30 p.m.

National Conference-Detroit: Saturday, Feb. 15, 2:00-4:00 p.m.

President's Program-San Francisco, 1997: Saturday, Feb. 15, 2:00-5:30 p.m.; Monday, Feb. 17, 8:00-11:00 a.m.

President's Program-Washington, D.C., 1998: Friday, Feb. 14, 11:00 a.m.-12:30 p.m.

President's Program Discussion Session: Monday, Feb. 17, 9:30-11:00 a.m. Topic: "New Leaming Communities: The Librarian's Role"

Professional Development: Sunday, Feh. 16, 8:00-9:00 a.m.; Monday, Feb. 17, 2:00-4:00 p.m. 
Professional Enhancement: Saturday, Feb. 15, 9:30-11:00 a.m.

Publications: Saturday, Feb. 15, 8:30-11:00 a.m.; Monday, Feb. 17, 8:00 a.m.-12:30 p.m.

Racial and Ethnic Diversity: Saturday, Feb. 15, 9:30 a.m.-12:30 p.m.; Coalition: Saturday, Feb. 15, 2:00-4:00 p.m.

Research: Saturday, Feb. 15, 9:30-11:00 a.m.; Sunday, Feb. 16, 9:30-11:00 a.m.

Standards and Accreditation: Monday, Feb. 17, 2:00-4:00 p.m.

Standards Study Task Force: Sunday, Feb. 16, 9:30-11:00 a.m.; Monday, Feb. 17, 2:00-4:00 p.m.

Statistics Committee: Sunday, Feb. 16, 9:30-11:00 a.m.; Monday, Feb. 17, 9:30-11:00 a.m.

\section{ACRL Chapters Council}

ACRL Presidential Candidates Forum/Re ception: Saturday, Feb. 15, 12:30-2:00 p.m.

Business and Orientation: Sunday, Feb. 16, 8:00 a.m. $-11: 00$ p.m.

\section{ACRL Editorial Boards}

Choice: Sunday, Feb. 16, 8:30-11:00 a.m.

College \& Research Libraries: Saturday, Feb. 15, 4:30-5:30 p.m.

College \& Research Libraries News: Sunday, Feb. 16, 9:30 a.m.-12:30 p.m.

New Publications Advisory Board: Sunday, Feb. 16, 11:30 a.m.-12:30 p.m.

Publications in Librarianship: Saturday, Feb. 15, 11:30 a.m.-12:30 p.m.

\section{AAMES meeting change}

After 10 years of meeting on Sunday afternoons, the Asian, African, and Middle Eastern Section (AAMES) Executive Committees has switched its meeting times in 1997 (both Midwinter and Annual) to Saturday afternoon, 2:00-4:00 p.m. This change was made with the hope that section members unable to attend the Sunday afternoon meetings will be able to come on Saturday. All are welcome at AAMES Executive Committee meetings who share an interest in issues related to academic libraries and Asian, African, and Middle Eastern area studies.
Rare Books \& Manuscripts Librarianship: Saturday, Feb. 15, 4:30-5:30 p.m.

\section{ACRL Sections}

Activity Sections Council: Friday, Feb. 14 , 4:30-5:30 p.m.

\section{Afro-American Studies Librarian \\ Section}

Executive: Saturday, Feb. 15, 9:30-11:00 a.m.

All Standing Committees: Saturday, Feb. 15, 8:00-9:00 a.m.

Business/Membership: Saturday, Feb. 15 2:00-4:00 p.m.

\section{Asian, African and Middle Eastern Section}

Executive and All Committees: Saturday, Feb. 15, 2:00-4:00 p.m.

\section{Anthropology and Sociology Section}

Executive: Saturday, Feb. 15, 9:30-11:00 a.m.; Monday, Feb. 17, 2:00-4:00 p.m.

Anthropology Librarians Discussion Group: Saturday, Fel). 15, 4:30-5:30 p.m.

Bibliography: Saturday, Feb. 15, 2:00-4:00 p.m.; Monday, Feb. 17, 8:30-11:00 a.m.

Conference Program Planning-San Francisco, 1997: Sunday, Feb. 16, 8:00-9:00 a.m.

Conference Program Planning-Washington, 1).C., 1998: Saturday, Feb. 15, 8:00-9:00 a.m.

Liaison: Sunday, Feb. 16, 9:30 a.m.-12:30 p.m.

Publications: Sunday, Feb. 16, 2:00-4:00 p.m.

Review and Planning: Sunday, Feb. 16, 4:30-5:30 p.m.

Sociology Librarians Discussion Group: Sunday, Feb. 16, 4:30-5:30 p.m.

\section{Arts Section}

Executive and Membership: Sunday, Feb. 16, 9:30 a.m. $-12: 30$ p.m.

All Committees: Saturday, Feb. 15, 9:30 a.m. $-12: 30$ p.m.

Dance Librarians Discussion Group: Monday, Feb. 17, 2:00-4:00 p.m.

Film and Broadcast Studies Discussion Group: Sunday, Feb. 16, 2:00-4:00 p.m.

Technology in the Arts: Monday, Feb. 17, 8:30-11:00 a.m

\section{Community and Junior College Libraries Section}

Executive: Saturday, Feb. 15, 11:30 a.m.-12:30 p.m.; Monday, Feb. 17, 9:30-11:00 a.m. 
All Committees: Saturday, Feb. 15, 2:00-4:00 p.m.

Awards: Sunday, Feb. 16, 8:30-11:00 a.m. (closed)

CJCLS/NCRL Joint Discussion Group: Sunday, Feb. 16, 2:00-4:00 p.m.

Conference Program Planning-San Francisco, 1997: Sunday, Feb. 16, 8:30-11:00 a.m.

Conference Program Planning-Washington, D.C., 1998: Sunday, Feb. 16, 9:30-11:00 a.m.

\section{College Libraries Section}

Executive: Saturday, Feb. 15, 9:30 a.m.-11:00 a.m.; Tuesday, Feb. 18, 9:30 a.m.-12:30 p.m.

CLIP Notes: Saturday, Feb. 15, 9:30-11:00 a.m.

College Libraries Discussion Group: Sunday Feb. 16, 11:30 a.m.-12:30 p.m.

College Library Directors' Discussion Group: Sunday, Feb. 16, 2:00-4:00 p.m.

College Library Leadership: Monday, Feb. 17, 8:00-9:00 a.m.

Communications: Saturday, Feb. 15, 2:00-4:00 p.m.

Conference Program Planning - San Francisco, 1997: Saturday, Feb. 15, 11:30 a.m.-12:30 p.m.

Conference Program Planning-Washington, D.C., 1998: Saturday, Feb. 15, 11:30 a.m.-12:30 p.m.

Continuing Education: Monday, Feb. 17, 9:30 a.m. $-12: 30$ p.m.

Medium-Sized Academic Libraries Discussion Group: Monday, Feb. 17, 2:00-4:00 p.m.

\section{Meet the candidates for ACRL president}

Hear Maureen Sullivan and James F. Williams III, the official candidates for ACRL vice-president/president-elect, speak at the ACRL Presidential Candidates Forum on Saturday, Feb. 15, 1997, 12:30-2:00 p.m., during the ALA Midwinter Meeting in Washington. Sullivan is an organizational development consultant. Williams is dean of libraries at the University of Colorado at Boulder. The forum, organized by the ACRL Chapters Council, is followed by a reception sponsored by EBSCO Subscription Services. Ed note: Seep. 738 for brief biograpbical sketches of the candidates.
Medium-Sized Academic Libraries Steering Committee: Saturday, Feb. 15, 8:00-9:00 a.m.

Research for College Librarianship: Sunday, Feb. 16, 9:30 a.m.-12:30 p.m.

Standards: Saturday, Feb. 15, 11:30 a.m. -12:30 p.m.; Monday, Feb. 17, 9:30-11:00 a.m.

\section{English and American Literature Section}

Executive: Saturday, Feb. 15, 2:00-4:00 p.m. General Membership Meeting: Sunday, Feb. 16, 9:30 a.m.-12:30 p.m.

All Committees: Monday, Feb. 17, 9:30 a.m. $-12: 30$ p.m.

\section{Education and Behavioral Sciences Section}

Executive: Friday, Feb. 14, 8:00-10:00 p.m.

Advisory Board: Sunday, Feb. 16, 4:30-7:00 p.m.

Conference Program Planning-Washington, DC, 1998: Saturday, Feb. 15, 4:30-5:30 p.m.

Curriculum Materials: Saturday, Feb. 15, 4:30-5:30 p.m.

Curriculum Materials Centers Management Saturday, Feb. 15, 4:30-5:30 p.m.

Government Policy: Saturday, Feb. 15, 4:30-5:30 p.m

Library Instruction for Educators: Saturday, Feb. 15, 4:30-5:30 p.m.

Membership and Orientation: Saturday, Feb. 15, 4:30-5:30 p.m.

Nominating 1998: Saturday, Feb. 15, 11:30 a.m. $-12: 30$ p.m.

Psychology and Psychiatry: Saturday, Feb. 15, 4:30-5:30 p.m.

Publications: Saturday, Feb. 15, 8:00-9:00 a.m.

Reference Services: Saturday, Feb. 15 4:30-5:30 p.m.

Social Work/Social Welfare: Saturday, Feb. 15, 4:30-5:30 p.m.

\section{Extended Campus Library Services Section}

Executive: Saturday, Feb. 15, 2:00-4:00 p.m.; Monday, Feb. 17, 11:30 a.m.-12:30 p.m.

All Committees: Sunday, Feb. 16, 8:30-11:00 a.m.

Discussion Group: Monday, Feb. 17, 8:30-11:00 a.m.

Planning: Monday, Feb. 17, 2:00-4:00 p.m.

Telecommunications Task Force: Sunday, Feb. 16, 11:30 a.m.-12:30 p.m. 


\section{Instruction Section}

Executive: Friday, Feb. 14, 9:30 a.m.-12:30 p.m.; Tuesday, Feb. 18, 9:30-11:00 a.m.

Active Learning Task Force: Saturday, Feb. 15, 2:00-4:00 p.m.; Sunday, Feb. 16, 9:30-11:0 a.m.

Advisory Council: Saturday, Feb. 15 9:30-11:00 a.m.; Monday, Feb. 17, 2:00-4:00 p.m

Awards: Saturday, Feb. 15, 2:00-5:30 p.m.; Monday, Feb. 17, 9:30 a.m.-12:30 p.m. (closed)

Communications: Saturday, Feb. 15, 11:30 a.m.-12:30 p.m.; Monday, Feb. 17, 9:30-11:00 a.m.

Communications Intern Orientation: Saturday, Feb. 15, 8:00-9:00 a.m.

Conference Program Planning-San Francisco, 1997: Saturday, Feb. 15, 2:00-4:00 p.m.
Conference Program Planning-Washington, D.C., 1998: Sunday, Feb. 16, 9:30-11:00 a.m. Monday, Feb. 17, 9:30-11:00 a.m.

Continuing Education: Sunday, Feb. 16, 9:30-11:00 a.m.; Monday, Feb. 17, 9:30-11:00 a.m.

Discussion Forum: Sunday, Feb. 16, 4:30-5:30 p.m.

Education for Library Instructors: Friday, Feb. 14, 4:30-5:30 p.m.; Sunday, Feb. 16, 9:30-11:00 a.m.; Monday, Feb. 17, 9:30-11:00 a.m.

Emerging Technologies in Instruction: Saturday, Feb. 15, 2:00-4:00 p.m.; Monday, Feb. 17, 9:30-11:00 a.m.

Glossary Projects Task Force: Saturday, Feb. 15, 2:00-4:00 p.m

\section{ACRL Discussion Groups}

Ed. Note: Section discussion groups are listed with their sections.

Alliances for New Directions in Teaching/Learning: Monday, Feb. 17, 8:00-9:00 a.m. Topic: "Lessons Learned: Librarians and Campus Teacbing and Learming Programs"

Australian Studies: Sundlay, Feb. 16, 11:30 a.m.-12:30 p.m. Topic: "Distance Is No Longer Isolation: The Internet Speeds the Change"

Canadian Studies: Sunday, Feb. 16, 11:30 a.m.-12:30 p.m. Topic: "Canadian Government Publications Depository Program/Electronic Publications Pilot Program"

Electronic Iibrary Development in Academic Libraries: Sunday, Feb. 16, 9:30-11:00 a.m. Topic: "Challenges in Merging Libraries and Computing Centers"

Electronic Reserves: Sunday, Feb. 16, 8:30-11:00 a.m. Topic: "Product demonstrations and an update on guidelines for fair use and electronic resernes

Electronic Text Centers: Saturday, Feb. 15, 2:00-4:00 p.m. Topic: "Cooperation in a Competitive World: Interinstitutional Approaches to Building and Senricing Electronic Text Collections"

Exhibits and Displays in College Libraries: Monday, Feb. 17, 2:00-4:00 p.m.

Fee-based Information Service Centers in Academic Libraries (FISCAL): Sunday, Feb, 16, 9:30-11:00 a.m. Topic: "Fifteen Years of FISCAL-Past, Present, and Future." "Database Management Systems, " and "Ready' Reference at Los Angeles County Public"
Fund-Raising \& Development: Sunday, Feb. 16, 9:30-11:00 a.m. Topic: "Future of Funding"

Heads of Public/Readers Services: Sunday, Feb. 16, 4:30-5:30 p.m.

Home Economics/Human Ecology Librarians: Sunday, Feb. 16, 11:30 a.m.-12:30 p.m. Topic: "Wby Didn't I Find This Article? Working Tou'ards Improved Indexing for Journals Related to Home Economics"

Institutional Priorities and Faculty Rewards: Saturday, Feb. 15, 2:00-4:00 p.m.

Librarians of Library Science Collections: Saturday, Feb. 15, 2:00-4:00 p.m.

MLA International Bibliography in Academic Libraries: Saturday, Feb. 15, 9:30 a.m.-12:30 p.m. Topic: "Interdisciplinary Searches in the MLA International Bibliography"

New Learning Communities: Monday, Feb. 17, 9:30-11:00 a.m. Topic: "New Leaming Communities: The Librarian's Role"

Personnel Administrators and Staff Development Officers of Large Research Libraries: Saturday, Feb. 15, 9:30 a.m.-12:30 p.m.; Sunday, Feb. 16, 9:30 a.m.-12:30 p.m.

Popular Culture and Libraries: Sunday, Feb. 16, 4:30-5:30 p.m. Topic: "The Internet and Popular Culture"

Public Relations in Academic Libraries: Monday, Feb. 17, 2:00-4:00 p.m. Topic: Library Exhibitions: Payoffs and Pitfalls"

Undergraduate Librarians: Monday, Feb. 17. 8:30-11:00 a.m. 
Instruction for Diverse Populations: Sunday, Feb. 16, 9:30-11:00 a.m.; Monday, Feb. 17, 9:30-11:00 a.m.

Knowledge Based Task Force: Saturday, Feb. 15, 2:00-4:00 p.m.

Management for Instruction Services: Sunday, Feb. 16, 9:30-11:00 a.m.; Monday, Feb. 17, 9:30-11:00 a.m.

Membership: Saturday, Feb. 15, 2:00-4:00 p.m.; Monday, Feb. 17, 9:30-11:00 a.m.

Nominating 1998: Sunday, Feb. 16, 11:30 a.m.-12:30 p.m.; Monday, Feh. 17, 11:30 a.m. $-12: 30$ p.m. (closed)

Planning: Saturday, Feb. 15, 2:00-4:00 p.m.; Monday, Feb. 17, 9:30-11:00 a.m.

Policy: Saturday, Feb. 15, 2:00-4:00 p.m.; Monday. Feb. 17, 9:30-11:00 a.m.

Preconference Planning-San Francisco, 1997: Saturday, Feb. 15, 2:00-4:00 p.m.; Sunday, Feb. 16, 9:30-11:00 a.m.

Strategic Plan Task Force: Sunday, Feb. 16, 9:30-11:00 a.m.

Teaching Methods: Sunday, Feb. 16, 11:30 a.m.-12:30 p.m.; Monday, Feb. 17, 9:30-11:00 a.m.

\section{Law and Political Sciences Section}

Executive and All Committees: Saturday, Feb. 15, 2:00-5:30 p.m.

Library Instruction: Sunday, Feb. 16, 2:00-4:00 p.m.

Marta Lange Award: Sunday, Feb. 16, 11:30 a.m. $-12: 30$ p.m.

\section{Rare Books and Manuscripts Section}

Executive: Sunday, Feb. 16, 2:00-4:00 p.m. Monday, Feb. 17, 8:30-11:00 a.m.

Bibliographic Standards: Saturday, Feb. 15 8:30-11:00 a.m.; Sunday, Feb. 16, 8:30-11:00 a.m.

Budget and Development: Saturday, Feb. 15, 2:00-4:00 p.m.

Conference Development: Sunday, Feb. 16, 8:30-11:00 a.m.

Conference Program Planning-San Francisco, 1997: Sunday, Feb. 16, 11:30 a.m.-12:30 p.m.

Conference Program Planning-Washington, 1998: Sunday, Feb. 16, 11:30 a.m.-12:30 p.m.

Curators and Conservators Discussion Group: Sunday, Feb. 16, 8:30-11:00 a.m.

Education and Professional Development: Saturday, Feb. 15, 2:00-4:00 p.m.

Exhibition Catalog Awards: Saturday, Feb. 15, 8:30 a.m-12:30 p.m.; Sunday, Feb. 16, 8:30 a.m-12:30 p.m (closed)
Information Exchange: Sunday, Feb. 16, 4:30-5:30 p.m.

Manuscripts and Other Formats Discussion Group: Saturday, Feb. 15, 2:00-4:00 p.m.

MARC for Special Collections Discussion Group: Sunday, Feb. 16, 11:30 a.m.-12:30 p.m.

Membership ad hoc: Saturday, Feb. 15, 8:00-10:00 p.m.

Nominating: Saturday, Feb. 15, 11:30 a.m. $-12: 30$ p.m.

Preconference Program Plànning-San Francisco, 1997: Saturday, Feb. 15, 8:30-11:00 a.m.

Preconference Program Planning-Washington, 1998: Saturday, Feh. 15, 11:30 a.m.-12:30 p.m.

Public Services Discussion Group: Sunday, Feb. 16, 8:30-11:00 a.m.

Publications: Saturday, Feb. 15, 2:00-5:30 p.m.

RBML Awards: Saturday, Feb. 15, 8:00-9:00 a.m. (closed)

Security: Saturday, Feb. 15, 8:30-11:00 a.m.

Seminars: Saturday, Feb. 15, 2:00-4:00 p.m.

\section{Slavic and East European Section}

Executive: Monday, Feb. 17, 11:30 a.m.-12:30 p.m.

Automated Bibliographic Control: Monday, Feb. 17, 9:30-11:00 a.m.

Conference Program Planning-San Francisco, 1997: Sunday, Feb. 16, 4:30-5:30 p.m.

Conference Program Planning-Washington. 1998: Monday, Feb. 17, 8:00-9:00 a.m.

Continuing Education: Sunday, Feb. 16, 9:30-11:00 a.m.

Newsletter: Sunday, Feb. 16, 11:30 a.m. $-12: 30$ p.m.

Preservation: Sunday, Feb. 16, 2:00-4:00 p.m.

\section{Address the ACRL Board}

Share your concerns directly with ACRL's Board of Directors. The first 21 minutes of the Sunday, Feb. 16, 1996, 2:00-4:00 p.m. meeting of the ACRL Board in Washington, D.C., will be given to an open-mike period. ACRL members may-on a first-come, firstserved basis-address the Board (with a limit of three minutes per speaker) on any topic. The Board hopes this communication method will facilitate hearing the concerns and interests of ACRL members. 


\section{Science and Technology Section}

Comparison of Science and Technology Libraries: Saturday, Feb. 15, 2:00-4:00 p.m.

Conference Program Planning-San Francisco, 1997: Sunday, Feb. 16, 9:30 a.m.-12:30 p.m.

Conference Program Planning-Washington, 1998: Sunday, Feb. 16, 9:30-12:30 p.m.

Continuing Education: Sunday, Feb. 16, 9:30 a.m. $-12: 30$ p.m.

Council: Friday, Feb. 14, 8:00-10:00 p.m.; Monday, Feb. 17, 8:30-11:00 a.m.

Discussion Group Leaders: Saturday, Feb. 15, 9:30 a.m. $-12: 30$ p.m.

Forum for Science and Technology Library Research: Sunday, Feb. 16, 8:30-11:00 a.m

General Discussion Group: Saturday, Feb. 15, 2:00-4:00 p.m. Topic: "Dealing with the Budget Crunch: Options for Acquisitions and Collection Development"

Heads of Science and Technology Libraries Discussion Group: Sunday, Feb. 16, 8:00-10:00 p.m. Topic: "Science Materials Budgets: How are They Formulated and How We are Advocates"

Legislation: Saturday, Feb. 15, 8:0010:00 p.m.

Membership and Recruitment: Saturday, Feb. 15, 9:30 a.m.-12:30 p.m.

Oberly Award: Saturday, Feb. 15, 9:30 a.m.-12:30 p.m. (closed)

Organization and Planning: Sunday, Feb. 16, 9:30 a.m.-12:30 p.m.

Publications: Saturday, Feb. 15, 9:30 a.m.-12:30 p.m.

Publisher/Vendor Relations Discussion Group: Saturday, Feb. 15, 9:30-11:00 a.m.

Science and Technology Databases Discussion Group: Sunday, Feb. 16, 11:30 a.m.-12:30 p.m. Topic: Science Materials Budgets: How are they Formulated and How We are Advocates"

Subject and Bibliographic Access: Saturday, Feb. 15, 9:30 a.m.-12:30 p.m.

\section{University Libraries Section}

Executive: Saturday, Feb. 15, 9:30-11:00 a.m.; Monday, Feb. 17, 9:30-11:00 a.m

Communications: Saturday, Feb. 15, 11:30 a.m. $-12: 30$ p.m.; Tuesday, Feb. 18, 9:30-11:00 a.m.

Conference Program Planning-San Francisco, 1997: Saturday, Feb. 15, 11:30 a.m.-12:30 p.m.; Monday, Feb. 17, 11:30 a.m. $-12: 30$ p.m.
Conference Program Planning-Washington, 1998: Saturday, Feb. 15, 11:30 a.m.-12:30 p.m.; Monday, Feb. 17, 11:30 a.m.-12:30 p.m.

Current Topics: Saturday, Feb. 15, 2:00-4:00 p.m.

Librarians in Higher Education Discussion Group: Sunday, Feb. 16, 9:30-11:00 a.m.

Nominating: Saturday, Feb. 15, 8:009:00 a.m. (closed)

Organization and Bylaws: Sunday, Feb. 16, 11:30 a.m.-12:30 p.m

Policy and Planning: Sunday, Feb. 16, 9:30-11:00 a.m.

Public Service Directors of Large Research Libraries Discussion Group: Sunday, Feb. 16, 2:00-4:00 p.m.

\section{Western European Specialists Section}

Executive: Tuesday, Feb. 18, 11:30 a.m.-12:30 p.m.

Classical, Medieval, and Renaissance Discussion Group: Saturday, Feb. 15, 2:00-4:00 p.m.

College and Medium-Sized Libraries Discussion Group: Monday, Feb. 17, 8:00-9:00 a.m.

Conference Program Planning-Washington, 1998: Saturday, Feb. 15, 2:00-4:00 p.m.

General Membership Meeting and Discussion Group: Monday, Feb. 17, 9:30-11:00 a.m. Topic: "European Union Electronic Information"

Germanists Discussion Group: Sunday, Feb. 16, 9:30-11:00 a.m.

Nijhoff Award Committee: Sunday, Feb. 16, 4:30-5:30 p.m. (closed)

Preconference Planning Committee-San Francisco, 1997: Saturday, Feb. 15, 8:00-10:00 p.m.

Publications: Tuesday, Feb. 18, 9:30-11:00 a.m.

Research and Planning: Sunday, Feb. 16, 2:00-4:00 p.m.

Romance Languages Discussion Group: Monday, Feb. 17, 2:00-4:00 p.m.

Scandinavian Discussion Group: Sunday, Feb. 16, 4:30-5:30 p.m.

\section{Women's Studies Section}

Executive: Monday, Feb. 17, 2:00-4:00 p.m.

All Committees: Sunday, Feb. 16, 9:30 a.m.-12:30 p.m.

Discussion Meeting: Monday, Feb. 17, 9:30-11:00 a.m. Topic: "Sorting out the New Women's Studies CD-ROM's"

General Membership: Sunday, Feb. 16, 4:30-5:30 p.m. 